\title{
Fetal thymus size evaluation using two-dimensional ultrasound in co-relation to fetal biometry as a sensitive parameter change in pregnancies complicated with fetal growth restriction (FGR)
}

Mahmoud Thabet',

Lamiaa El-Sorougy ${ }^{2}$

'Department of Obstetrics and

Gynecology, Mansoura Faculty

of Medicine, Mansoura University;

Egypt.

2Department of Diagnostic

Radiology, Mansoura Faculty of Medicine, Mansoura University; Egypt.
Mahmoud Thabet

Lecturer of Obstetrics and

Gynecology Mansoura Faculty of

Medicine, Mansoura University,

Egypt

Email: thabet0777@gmail.com

Tel: 01003398201

\begin{abstract}
Objective: Fetal thymus size evaluation through measuring its ultrasonographic maximum transverse diameter, in relation to fetal biometry changes in pregnancies complicated with FGR.

Methods: Our study included 66 singleton pregnancies with intact membranes, aged (18 to $40 \mathrm{y}$ ) between 28 and 36 weeks of gestation with FGR,Patients with fetal or maternal infections, chromosomal or fetal congenital anomalies, or IUFD and patients start labor were excluded. All patientsunderwentultrasound evaluation weekly during the third trimester after history taking; examination and lab investigation were performed, where measurements of themaximum transverse diameter of the fetal thymus and fetal biometry (BPD percentile,FL percentile, AC percentile,EFW percentile,and Single deepest vertical pocket) were obtained. The small thymus was defined as a thymus perimeter $\leq 5^{\text {th }}$ percentile according to a fetal thymus nomogram, then Patients were classified into 2 groupsaccording to whether they had a small transverse thymus diameter $\left(<5^{\text {th }} \%\right)$ or normal transverse thymus diameter $\left(>5^{\text {th }} \%\right)$. The co-relation between fetal thymus size, obstetric history and fetal biometry changes by ultrasonography were performed.
\end{abstract}

Results: The sixty-six FGR pregnancies were classified to 55 patients with thymus diameter less than $5^{\text {th }}$ percentile and 11 patients with thymus diameter more than $5^{\text {th }}$ percentile. The co-relation between thymus diameter less and more than $5^{\text {th }}$ percentile and obstetric history showed that no statistically significant difference as regarding history of abortion, history of stillbirth, history of preterm labor and history of IUGR but there was statistically significant difference as regarding gestational age (GA) mean at enrollment (32.04 \pm 2.7 vs. $36.5 \pm 1.04)$ meaning IUGR fetuses with a thymus diameter $<5^{\text {th }} \%$ presented lower GA at enrollment. The correlation between thymus diameter and fetal biometry shows a highly significant positive correlation between thymus transverse diameter and FL, AC, EFW, and AF vertical pocket but non-significant correlation as regards $\mathrm{BPD}$ in all cases of both groups.

The comparison between thymus diameters less and more than $5^{\text {th }}$ percentile in relation to fetal ultrasonic biometry showed that no statistically significant difference as regarding BPD mean percentile and FL mean percentile, on other hand, AC and EFW mean percentiles were statistically significantly higher among IUGR fetuses with normal thymus diameter $\left(>5^{\text {th }}\right.$ percentile) $[3.2 \pm 2.7$ vs. $6.6 \pm 2.2$ $\& 3.06 \pm 2.6$ vs. $6.09 \pm 2.5$ ]. $90.91 \%$ of IUGR fetuses with normal thymus diameter $\left(>5^{\text {th }}\right.$ percentile) have adequate amniotic fluid as 
estimated by single deepest vertical pocket versus $34.55 \%$ of IUGR fetuses with thymus diameter $<5^{\text {th }}$ percentile with a statistically significant difference. All IUGR fetuses with oligohydramnios $(n=20)$ had small thymus $<5^{\text {th }} \%$.

Conclusion: There is a highlypositive correlation between thymus transversediameter and AC, EFW, and AF vertical pocket inpregnancies complicated with FGR.

Keywords: Fetal thymus size, fetal growth restriction,obstetric history, and fetal biometry.

\section{Introduction}

Fetal growth reduction is seen in about $10 \%$ of the pregnancies. Few of them have a pathological background known as fetal growth restriction (FGR). FGR is defined as pathologicalinhibition of fetal intrauterine growth $\mathrm{s}$ and the failure to achieve its growth potential. Predictive parameters founding were tried by research in this field, aiming to reach an early diagnosis, which would lead to a better management of the condition. ${ }^{1}$

The estimation of fetal weightusing ultrasonic measurements of the abdominal circumference (AC), head circumference (HC), biparietal diameter (BPD), and femur length (FL), investigators developed mathematical formulas and constricted percentile nomograms of estimation of fetal weight $(\mathrm{EFW})$ at different gestational ages. The most commonly used equations and nomograms are those of Shepard et al, (1982) ${ }^{2}$, and Hadlock et al. $(1985)^{3}$, and one or both of these nomograms were incorporated into software of most ultrasound machines.

United States diagnosis of FGR depend on estimation of fetal weight (EFW), where it measure below 10th percentile through utilize measurements of BPD (biparietal diameter), FL (femur length), AC (abdominal circumference) and HC (head circumference). ${ }^{4}$

One of a common finding in FGR isOligohydramnios. The FGR incidence was 5\% when the amniotic fluid volume was normal but when oligohydramnios was present it increased to approximately $40 \% .^{5}$

Thymus presents in the upper part of the chest cavity, formed of two-lobed structure that extends partially into the neck region. It is situated above the pericardium of the heart, between the lungs, anterior to the aorta, below the thyroid, and behind of the sternum. ${ }^{6}$

Themeasurement ofthe transverse diameter of the thymusis easier than that of the perimeter due to its interface with the lungs, that demarcating the lateral margins of the thymus. Thethymus transverse diameter can be defined more consistently and is therefore readily measurable. ${ }^{7}$

Thetransverse diameter of fetal thymus increased in a linear manner in relation to the fetal GA, BPD, FL and AC.6

There was a relationship between the thymus transverse diameter and the gestational age, femoral length,and fetal abdominal circumference. The average transverse thymus diameter in $\mathrm{mm}$ was similar to the $\mathrm{AC}$ in $\mathrm{cm}^{7}$

\section{METHODS}

This is a prospectivecross-sectional clinical study that was carried out during the period between January 2017 and January 2018,conducted in the Antenatal clinic and The Mansoura University Hospital Antenatal Department. The study was approved by the local Institutional Research Ethical Committee "institutional research board". Total of 66 patients aged (18 to $40 \mathrm{y}$ ) between 28 and 36 weeks of gestation, singleton, accurate estimation of gestational age, with intact membranes and non -malformed FGR fetuses were included.

A written consent was given for all participants before being included and after explaining the study with the patient's ability to be withdrawn at any time under her own will.

All the studied patients were subjected to complete history taking, clinical examination, laboratory investigations, and ultrasound examination: use a trans-abdominal 3.5-MHz convex electronic probe(Logic p-52012). The fetal position, presentation, viability, amniotic fluid index and grade and site of placenta were first evaluated. Then, the biometric indices which include: head circumference (HC), abdominal circumference (AC), expected fetal weight (EFW), biparietal diameter (BPD), andfemur length (FL) which is the most useful measurement for assessing possible FGR were evaluated. 
Fetal thymus maximum transverse diameter was measured on a transverse sectionat the level of fetal chest anterior to three great vessels and posterior to the sternum, where we can measurethe thymus maximum transverse diameter through placing a line cursorin perpendicular to the line extending from the sternum to the spine. The mean of 3 measurements was used for the statistical study. Whenthe transverse thymus diameter showing a decrease below the $5^{\text {th }}$ percentile for gestational age refer to small fetal thymus according to the nomograms.

Patients were classified into 2 groups according to whether they had a small transverse thymus diameter $\left(<5^{\text {th }} \%\right)$ or normal transverse thymus diameter $\left(>5^{\text {th }} \%\right)$. The correlation between fetal thymus size by ultrasonography, obstetric history,andfetal biometry performed.

\section{Statistical analysis:}

The data collected were statistically analyzed byusing SPSS (statistical package for social sciences) statistical software version 21.0 (SPSS Inc., Chicago, IL,USA), Numerical data were described in terms of means with standard deviation, medians and range, minimum and maximum for dispersion. Percentages and frequencies (number of cases) were used when appropriate. Chi-square test was used to compare qualitative variables; $t$-tests were used to compare quantitative variables. Resulting data were presented in graphs, numeric and tabular forms. The comparison was considered significant when the probability of difference $(\mathrm{P}-\mathrm{value}) \leq 0.05$.

The comparison was considered highly significant when the probability of difference $(\mathrm{P}-\mathrm{value})<0.001$. If $\mathrm{P}$-value was more than $0.05(\mathrm{P}>0.05)$ refer tonon-significant differences. ${ }^{8}$

\section{RESULTS}

The 66pregnancies with FGR showed those 55 patients with fetal thymus diameter less than $5^{\text {th }}$ percentile and 11 patients with fetal thymus diameter more than $5^{\text {th }}$ percentile. The co-relation between thymus diameter less and more than $5^{\text {th }}$ percentile and obstetric history showed that no statistically significant difference as regarding History of abortion( $27.27 \%$ vs. $18.18 \%)$, History of stillbirth $(7.27 \%$ vs. $0 \%)$, History of preterm labor (18.18\% vs. $9.09 \%)$ and History of IUGR (16.36\% vs. $9.09 \%$ ) but there was statistically significant difference as regarding gestational age (GA) mean at enrollment $(32.04 \pm 2.7$ vs. $36.5 \pm 1.04)$ meaning IUGR fetuses with a thymus diameter $<5^{\text {th }} \%$ presented lower GA at enrollment as presented in table (1). The correlation between thymus diameter and fetal biometry shows a highly significant positive correlation between thymus transverse diameter and FL, AC, EFW, and AF vertical pocket but non-significant correlation as regards BPD in all cases of both groups which was represented in the table (2) and graph1, 2 and 3.

Table (3) showed the comparison between thymus diameters less and more than $5^{\text {th }}$ percentile in relation to fetal ultrasonic biometry showed that no statistically significant difference as regarding BPD mean percentile $(9.9 \pm 10.2$ vs. $14.9 \pm 12.9)$ and FL mean percentile $(3.9 \pm 3.11$ vs. $8 \pm 6.1)$, on other hand, AC and EFW mean percentiles were statistically significantly higher among IUGR fetuses with normal thymus diameter $\left(>5^{\text {th }}\right.$ percentile) $[3.2 \pm 2.7$ vs. $6.6 \pm 2.2 \& 3.06 \pm 2.6$ vs. $6.09 \pm$ 2.5]. $90.91 \%$ of IUGR fetuses with normal thymus diameter $\left(>5^{\text {th }}\right.$ percentile $)$ have adequate amniotic fluid as estimated by single deepest vertical pocket versus $34.55 \%$ of IUGR fetuses with thymus diameter $<5^{\text {th }}$ percentile with a statistically significant difference. All IUGR fetuses with oligohydramnios $(n=20)$ had small thymus $<5^{\text {th }} \%$.

\section{Discussion}

FGR detection sensitivity in high-risk patients usingEFWhas a range of $33.3 \%$ to $89.2 \%$ with specificity range of $53.7 \%$ to $90.9 \%$. Where AC below10th percentilesensitivity was $72.9 \%$ and specificities were $94.5 \%$ that optimize utilization of the EFW and AC percentiles in the diagnosis of pathological FGR. A normal AC associated with significantreduction in the incidence of FGR. ${ }^{9}$

Oligohydramnios is a common finding in FGR andamniotic fluid volumemeasurement is important forsurveillance of PFGR. Amniotic fluid volume can be assessed by measuring the maximum vertical pocket. The largest umbilical cord-free pocket of fluid with diameter $<2 \mathrm{~cm}$, Either an AFI $<5$ $\mathrm{Cm}$ or $<10$ th percentile for gestational age may be used to define oligohydramnios. ${ }^{5}$ 
In our study, the transverse diameter measurement of the thymus was possible. We found a statistically significant correlation between thymus diameter and GA, FL, AC, and EFW. There wasan agreement between our data as regards the thymus transverse diameter and that already presented by Cho et al study. ${ }^{7}$ They measure the maximum transverse diameter of the thymus in 352 normal fetuses between 19-38 weeks of gestation. They assessed the relationship of the transverse diameter with GA, $\mathrm{BPD}, \mathrm{FL}, \mathrm{AC}$, and $\mathrm{EFW}$ in a linear manner. ${ }^{7}$

The study of Cromi et al ${ }^{10}$ included 60 patients with FGR fetuses and 60 control group appropriate for -gestational age fetuses in two academic hospitals (University of Insubria and University of Verona), which revealed that proportion of fetuses with thymus perimeter $<5^{\text {tho }} \%$ for gestational age was significantly higher in FGR compared with controls (58/60 vs. 7/60). Similarly, in Olearo et al, ${ }^{11}$ studies revealed that fetal thymus volumes with birth weight andan abdominal circumference below the 10th percentile were significantly lower in comparison to healthy controls.

Our study comesconcomitant with Ekin et al ${ }^{12}$ study stated that ultrasonography IUGR evaluation revealed involution of the fetal thymus gland size. A small fetal thymus can bdconsidered as an early indicator of perinatal adverse outcomes. Also Yang et al, ${ }^{13}$ studiesusing 2D ultrasonography showed that fetal thymusanteroposterior andtransverse diameters and volume had increased with the normal advance of gestational weeks. In IUGR, these parameters were less than those of the same gestational age fetal thymus

\section{Conclusion}

Thymus gland size may be considered as a sensitive parameter for FGR changes, it has a highly positive correlation $\mathrm{AC}, \mathrm{EFW}$, and $\mathrm{AF}$ vertical pocket

\section{References}

1. American College of Obstetricians and Gynecologists. ACOG Practice bulletin no. 134: fetal growth restriction. Obstet Gynecol. 2013; 121(5):1122-33.

2. Shepard MJ, Richards VA, Berkowitz RL, Warsof SL, Hobbins JC. An evaluation of two equations for predicting fetal weight by ultrasound.
Am J Obstet Gynecol. 1982; 142(1):47-54.

3. Hadlock FP, Harrist R, Sharman RS, Deter RL, Park SK. Estimation of fetal weight with the use of head, body, and femur measurements - a prospective study. Am J Obstet Gynecol. 1985; 151(3):333-7.

4. Stirnemann J, Villar J, Salomon L, Ohuma E, Ruyan P, Altman D, et al. International estimated fetal weight standards of the INTERGROWTH-21st Project. J Ultrasound Obstet Gynecol. 2017; 49(4):478-86.

5. Madaan S, Mendiratta SL, Jain PK, Mittal M. Amniotic Fluid Index and its Correlation with Fetal Growth and Perinatal Outcome. J Fetal Med. 2015; 2(2):61-7.

6. Goldstein AL. History of the discovery of the thymosins. Ann NY Acad Sci. 2007; 1112(1):1-13.

7. Cho J, Min J, Lee YH, McCrindle B, Hornberger L, Yoo SJ. Diameter of the normal fetal thymus on ultrasound. Ultrasound Obstet Gynecol. 2007; 29(6):634-8.

8. Styan GP, Boyer C, Chu KL. Some comments on Latin squares and on Graeco-Latin squares, illustrated with postage stamps and old playing cards. Statistical Papers. 2009; 50(4):917-41.

9. Papageorghiou A, Kennedy S, Salomon L, Ohuma E, Ismail LC, Barros F, et al. International standards for early fetal size and pregnancy dating based on ultrasound measurement of crown-rump length in the first trimester of pregnancy. J Ultrasound Obstet Gynecol. 2014; 44(6):641-8.

10. Cromi A, Ghezzi F, Raffaelli R, Bergamini V, Siesto G, Bolis P. Ultrasonographic measurement of thymus size in IUGR fetuses: a marker of the fetal immunoendocrine response to malnutrition. Ultrasound Obstet Gynecol. 2009; 33(4):421-6.

11. Olearo E, Oberto M, Ogge G. Thymic volume in healthy, small for gestational age and growth restricted fetuses. Prenat Diagn 2012; 32:662-667.

12. Ekin A, Gezer C, Taner CE, Solmaz U, Gezer NS, et al. (2016) Prognostic Value of Fetal Thymus Size in Intrauterine Growth Restriction. J Ultrasound Med 35(3): 511-517.

13. Yang R, Guo F, Liu X, Tian J, Fan L. Application of two and three-dimensional ultrasound measurement of fetal thymus in fetaintrauterine growth restriction.Zhonghua Yi Xue Za Zhi. 2014 Sep 9; 94(33):2607-9. 
Table 1: Comparison between patients with thymus diameter less and more than $5^{\text {th }}$ percentile regarding obstetric history

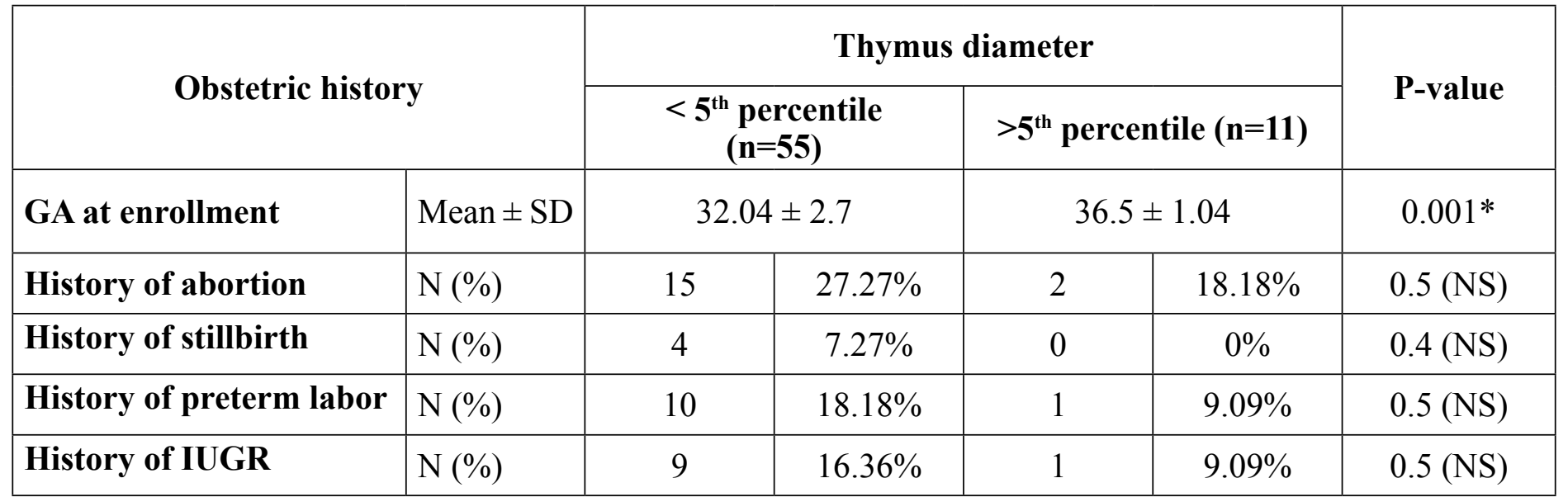

NS: no statistically significant difference*statistically significant difference

Table 2: Correlation between thymus diameter and fetal biometry

\begin{tabular}{|l|c|c|}
\hline \multirow{2}{*}{\multicolumn{1}{c}{ Fetal biometry }} & \multicolumn{2}{c|}{ Thymus diameter percentile } \\
\cline { 2 - 3 } & r & p-value \\
\hline BPD percentile & 0.2 & $0.2(\mathrm{NS})$ \\
\hline FL percentile & 0.4 & $0.002^{*}$ \\
\hline AC percentile & 0.4 & $0.002^{*}$ \\
\hline EFW percentile & 0.4 & $0.001^{*}$ \\
\hline Single AF deepest vertical pocket & 0.3 & $0.01^{*}$ \\
\hline
\end{tabular}

Graph 1: Correlation between thymus diameter and femur length

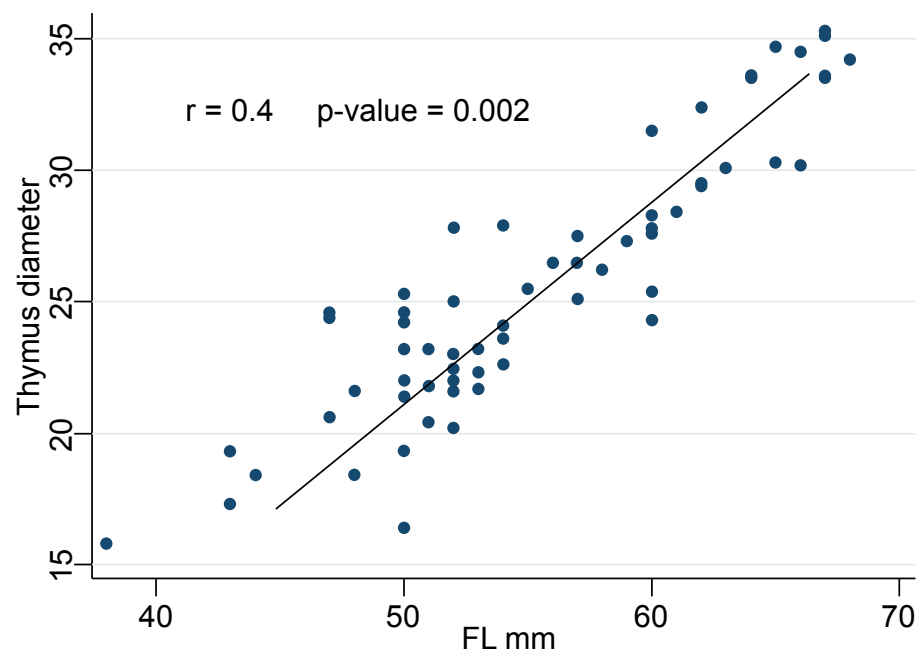

Thymus diameter plotted against femur length $(\mathrm{mm})$. The solid line represents the mean predicted from the regression equation. Dots represent actual data points. Regression equation thymus diameter $(\mathrm{cm})=$ $0.63 \times$ FL (cm)- 0.62 .
Graph 2: Correlation between thymus diameter and abdominal circumference

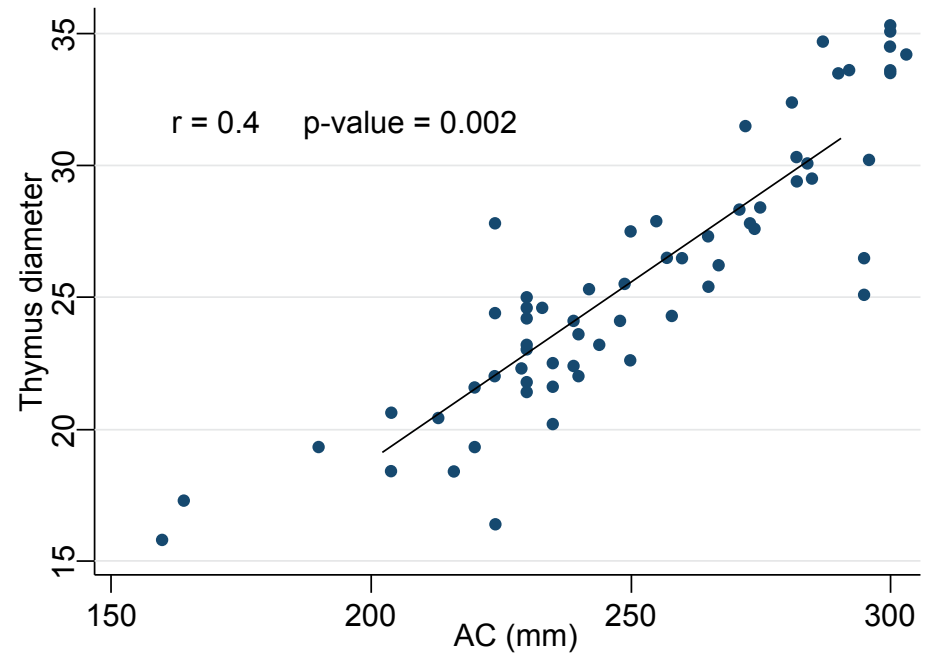

Thymus diameter plotted against AC. The solid line represents the mean predicted from the regression equation. Dots represent actual data points. Regression equation thymus diameter $(\mathrm{cm})==0.14 \times \mathrm{AC}(\mathrm{cm})$ 0.75 . 
Graph 3: Correlation between thymus diameter and estimated fetal weight

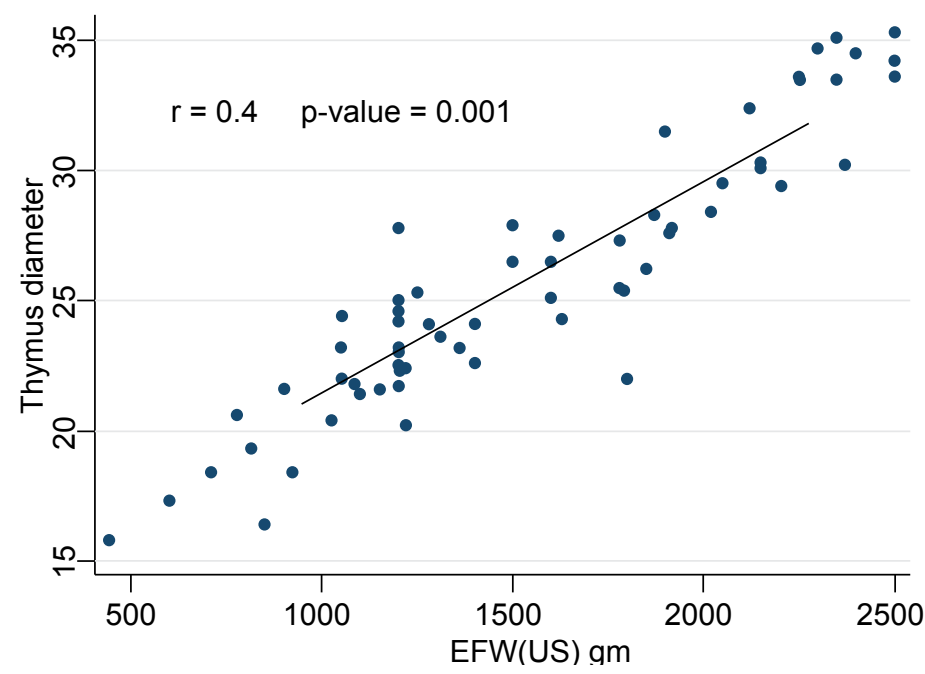

Thymus diameter plotted against EFW). Thesolid line represents the mean predicted from the regression equation. Dots represent actual data points

Table 3: Comparison between patients with thymus diameter less and more than $5^{\text {th }}$ percentile regarding fetal ultrasonic biometry

\begin{tabular}{|c|c|c|c|c|c|c|}
\hline \multirow{2}{*}{\multicolumn{2}{|c|}{ Fetal biometry }} & \multicolumn{4}{|c|}{ Thymus diameter } & \multirow{3}{*}{$\begin{array}{c}\text { P-value } \\
0.3(\mathrm{NS}) \\
\end{array}$} \\
\hline & & \multirow{2}{*}{\multicolumn{2}{|c|}{$\begin{array}{c}\begin{array}{c}\mathbf{5}^{\text {th }} \text { percentile } \\
(\mathbf{n}=\mathbf{5 5})\end{array} \\
9.9 \pm 10.2 \\
\end{array}$}} & \multirow{2}{*}{\multicolumn{2}{|c|}{$\begin{array}{c}\mathbf{P 5}^{\text {th }} \begin{array}{c}\text { percentile } \\
(\mathbf{n}=\mathbf{1 1})\end{array} \\
14.9 \pm 12.9\end{array}$}} & \\
\hline BPD percentile & Mean \pm SD & & & & & \\
\hline FL percentile & Mean \pm SD & \multicolumn{2}{|c|}{$3.9 \pm 3.11$} & \multicolumn{2}{|c|}{$8 \pm 6.1$} & $0.05(\mathrm{NS})$ \\
\hline AC percentile & Mean \pm SD & \multicolumn{2}{|c|}{$3.2 \pm 2.7$} & \multicolumn{2}{|c|}{$6.6 \pm 2.2$} & $0.003^{*}$ \\
\hline EFW percentile & Mean \pm SD & \multicolumn{2}{|c|}{$3.06 \pm 2.6$} & \multicolumn{2}{|c|}{$6.09 \pm 2.5$} & $0.002 *$ \\
\hline \multirow{3}{*}{$\begin{array}{l}\text { Single deepest } \\
\text { vertical pocket }\end{array}$} & Adequate & 19 & $34.55 \%$ & 10 & $90.91 \%$ & \multirow{3}{*}{$0.002 *$} \\
\hline & borderline & 16 & $29.09 \%$ & 1 & $9.09 \%$ & \\
\hline & oligohydramnios & 20 & $36.36 \%$ & 0 & $0 \%$ & \\
\hline
\end{tabular}

NS: no statistically significant difference*statistically significant difference 